\title{
Caractérisation des peuplements ligneux de la zone Cayor Baol (Thiès-Sénégal)
}

\author{
Minda MAHAMAT-SALEH, Aly DIALLO, Ndiaye OUSMANE, \\ Madiara Ngom FAYE et Aliou GUISSE * \\ Université Cheikh Anta Diop de Dakar, B.P 5005 Dakar, Sénégal. \\ *Auteur correspondant,E-mail: aliou.guisse@ucad.edu.sn; alguisse@orange.sn
}

Tel. $(+221) 776389669$

\section{RESUME}

Cette étude se propose de déterminer la composition floristique et la structure des peuplements ligneux dans trois localités de la région de Thiès (Fandène, Mont Rolland et Sessène). Sur un transect orienté Nord/Sud, partant de Mont Rolland à Sessène, un inventaire floristique et des relevés dendrométriques ont été effectués en fonction des unités morphopédologiques. Le traitement statistique des données recueillies a montré que la flore ligneuse, dans ces 3 sites, est riche de 35 espèces réparties en 30 genres appartenant à 20 familles. La famille des Mimosaceae est la mieux représentée, suivie des Caesalpiniaceae, des Combretaceae et des Anacardiaceae. La richesse floristique varie suivant les sites. La surface terrière est plus élevée au Mont Rolland, le recouvrement et la densité sont plus importants à Fandène. La distribution du peuplement selon la circonférence est homogène à Fandène, contrairement à Sessène et à Mont Rolland. Quant à la distribution selon la hauteur des individus, elle montre une prédominance de la strate arbustive dans les 3 sites. Les analyses de régression ont montré une corrélation entre la circonférence et la hauteur. La pression anthropique sur la strate ligneuse est plus importante à Sessène, moyenne au Mont Rolland et faible à Fandène. L'AFC montre une forte hétérogénéité des peuplements ligneux. Elle a révélé l'existence de 3 groupements en étroite relation avec les facteurs édaphologiques.

(C) 2013 International Formulae Group. All rights reserved.

Mot clés : Thiès, ligneux, flore, structure, groupement.

\section{INTRODUCTION}

Dans les zones sahélo-soudaniennes au Sénégal, les écosystèmes subissent une forte dégradation en raison de la péjoration climatique et des actions anthropiques (Ndiaye, 2009 ; Bakhoum, 2012). Ces effets négatifs conduisent à la réduction de la fertilité et du rendement des terres (INP, 2009).
La région de Thiès n'est pas épargnée par ces phénomènes de dégradation. En effet, ces derniers sont liés à l'érosion, à la salinisation et surtout aux actions anthropiques à travers le surpâturage et les feux de brousse (Bakhoum, 2012). Nos recherches bibliographiques ne nous ont pas permis d'avoir des résultats exhaustifs sur la végétation ligneuse dans cette zone. Ainsi, la caractérisation de la végétation s'avère 
nécessaire pour connaître l'état actuel des peuplements ligneux et leur niveau de dégradation, afin de proposer des stratégies de gestion et de réhabilitation de ces écosystèmes.

C'est dans ce contexte que notre étude s'intègre, avec comme objectif général la caractérisation de la flore et végétation ligneuse de la région de Thiès. Il s'agira d'établir la liste floristique ainsi que la structure des peuplements ligneux, d'identifier les groupements végétaux et d'apprécier la régénération naturelle.

Ce travail se fonde sur l'hypothèse selon laquelle les trois sites (Fandène, Mont Rolland et Sessène) n'ont pas échappé à cette dégradation qui se manifeste par une modification de la composition floristique, de la structure des peuplements ligneux et par une faible régénération naturelle des espèces ligneuses.

\section{MATERIEL ET METHODES}

\section{Présentation de la zone d'étude}

Avec une superficie de $6601 \mathrm{~km}^{2}$ (CSE, 2009), la région de Thiès fait partie intégrante du bassin arachidier sénégalais situé au centre du pays.

Le climat est du type soudano-sahélien (Aubréville, 1962), avec deux saisons : une saison pluvieuse de juin à octobre et une saison sèche de novembre à mai. Les températures moyennes tournent autour de 29 ${ }^{\circ} \mathrm{C}$. La pluviométrie moyenne annuelle (1999 - 2009) est de 484,5 mm.

Les sols correspondent aux sols ferrugineux tropicaux lessivés. Ils sont caractérisés par une texture sableuse et occupent près de $70 \%$ des sols de la région de Thiès. On y trouve aussi les sols ferrugineux tropicaux peu ou pas lessivés dominés par l'argile et représentant $25 \%$ des sols. Enfin, les sols hydromorphes appelés sols de bas fonds caractérisés par une texture argilohumifère occupent 5\% des sols (INP, 2009).
Trois sites (Mont Rolland, Fandène et Sessène) ont été utilisés comme base d'échantillonnage. Leur choix a été motivé par un souci de couvrir les limites géographiques (du nord au sud) et les différentes unités pédologiques rencontrées dans la zone d'étude (Figure 1).

Mont Rolland, situé au nord de la région de Thiès, est caractérisé par les sols argileux et par Adansonia digitata L. comme espèce dominante. Fandène, situé au centre, est caractérisé par les sols sableux et par l'abondance de Borassus aethiopium Mart (Thione, 2000). Et enfin Sessène, situé au sud, est caractérisé par des sols argilo-sableux et par Combretum glutinosum Perr.ex. DC comme espèce dominante (Minda, 2009).

\section{Méthodes \\ Collecte des données}

$\mathrm{Au}$ total 69 relevés dendrométriques, de $2500 \mathrm{~m}^{2}$ chacun, ont été effectués. Ces placettes sont réparties de manière aléatoire dans chaque site en fonction des différentes unités morphopédologiques: 25 placettes à Fandène, 22 à Mont Rolland et 22 à Sessène. La liste floristique a été établie sur la base de la Flore du Sénégal (Berhaut, 1967) et des arbres et arbustes du Sahel (Maydell, 1983). Les synonymes ont été actualisés et normalisés sur la base de l'Enumération des Plantes à Fleurs d'Afrique Tropicale (Lebrun et stork, 1997).

Les mensurations ont concerné uniquement les individus à circonférence basale supérieure ou égale à $10 \mathrm{~cm}$. La circonférence du tronc à $30 \mathrm{~cm}$ du sol et les diamètres du houppier (Est/Ouest et Nord/Sud) ont été mesurés pour établir le recouvrement et la surface terrière. La hauteur totale a été mesurée pour établir la structure du peuplement. Les analyses de régression ont été effectuées pour déterminer la relation entre circonférence et hauteur et la dominance relative. 


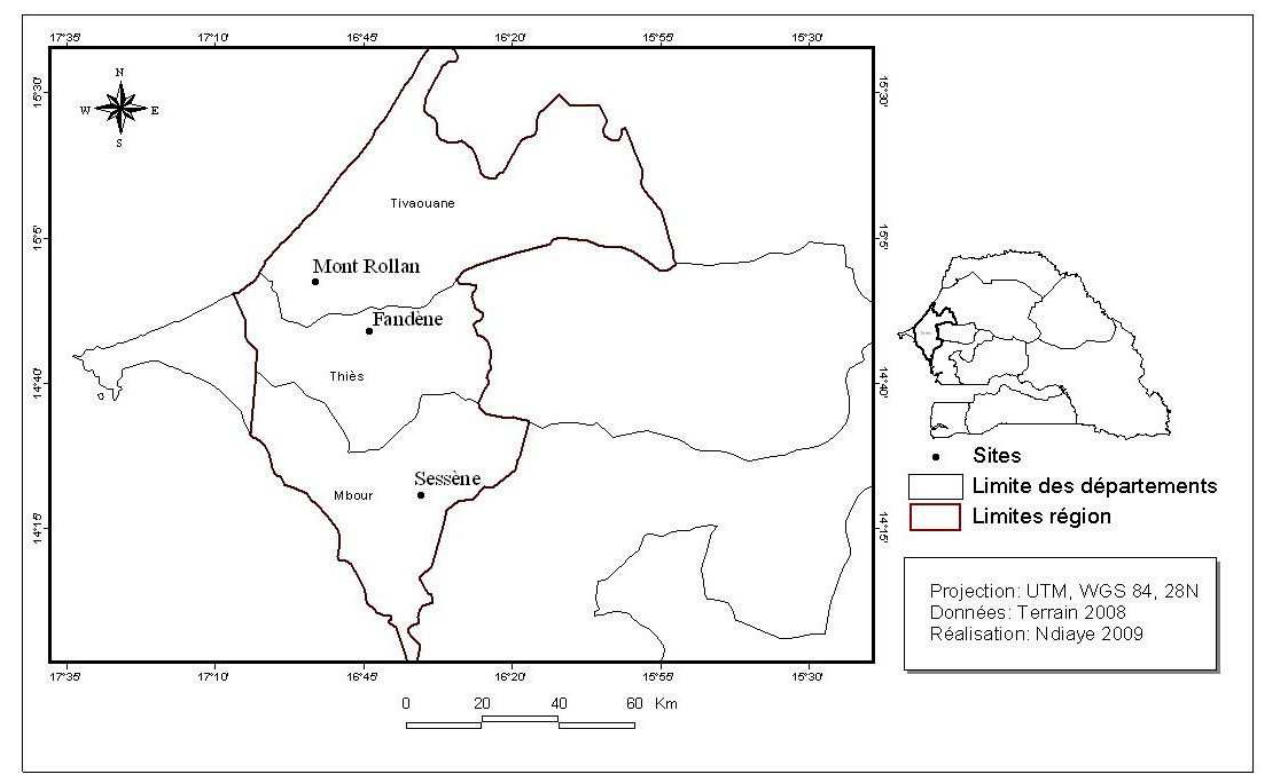

Figure 1 : Situation des sites d'étude.

\section{Traitement des données}

Les données recueillies ont été soumises à une analyse factorielle de correspondance (AFC) grâce au logiciel XLSTAT. Ces relevés nous ont permis de caractériser le couvert ligneux et de dégager l'état actuel des peuplements ligneux en calculant la densité, la surface terrière et le recouvrement.

Deux types de densité ont été déterminés :

La densité réelle nous a permis d'apprécier l'occupation de chacune des espèces dans le peuplement. Elle est égale au nombre d'individu par unité de surface (Nombre d'individus.ha ${ }^{-1}$ ). Quant à la densité théorique, elle est obtenue à partir de la distance moyenne $(\mathrm{m})$ entre les arbres en utilisant la relation de Wouters et Notelaers (1999):

$$
\text { Densité théorique }=\frac{10000}{(\mathrm{dm})^{2}}
$$

La surface terrière du peuplement est la somme des surfaces terrières de tous les ligneux qui constituent le peuplement. Elle est calculée pour les arbres et arbustes ayant une circonférence à la base du tronc à $0,30 \mathrm{~m} \mathrm{du}$ sol. La surface terrière se calcule par la formule de Rondeux (1993), puis rapportée à la surface échantillonnée $\left(\mathrm{ha}^{-1}\right)$.

$$
\mathrm{St}=\sum \frac{\mathrm{C}^{2}}{4 \pi}
$$

$\mathrm{St}=$ Surface terrière exprimée en $\mathrm{m}^{2} \cdot \mathrm{ha}^{-1}$

$\mathrm{C}=$ Circonférence à $30 \mathrm{~cm}$ du sol des individus mesurés en centimètre.

Quant au recouvrement, il est la somme des surfaces de la couronne de tous les individus de peuplement (Rondeux, 1993), il est aussi rapporté à la surface échantillonnée à l'hectare.

$$
\mathrm{Sc}=\sum \frac{\pi \times \mathrm{D}^{2}}{4}
$$

$\mathrm{S}_{\mathrm{C}}=$ Surface de la couronne exprimée en $\mathrm{m}^{2} \cdot \mathrm{ha}^{-1}$

$\mathrm{D}=$ Moyenne des diamètres Est/Ouest et Nord/Sud exprimée en mètre.

Le niveau d'anthropisation a été évalué à partir des individus coupés ou abattus. Cette 
anthropisation du peuplement ligneux a été calculée à partir du rapport exprimé en pourcentage du nombre de pieds exploités (coupes, abattage) de tous les individus divisés par le nombre total d'individus par site selon la formule ci-dessous:

Nombre de pieds exploités

$$
\text { AP = -------------------------- X } 100
$$

La régénération a été déterminée par dénombrement de l'ensemble des jeunes plants de chaque espèce. Le caractère juvénile est attribué à tout individu dont la circonférence à la base du tronc $(30 \mathrm{~cm} \mathrm{du}$ sol) est inférieure à $10 \mathrm{~cm}$ (Diallo et al., 2011).

\section{RESULTATS}

\section{Identification des groupements végétaux}

La matrice de 69 relevés x 35 espèces ligneuses a été soumise à l'analyse factorielle des correspondances pour définir la variation spatiale du peuplement ligneux. Les valeurs provenant du Tableau 1 ont permis d'interpréter le résultat de l'analyse.

Les deux premiers axes $\left(\mathrm{F}_{1}\right.$ et $\left.\mathrm{F}_{2}\right)$ définissent le plan principal de l'analyse, ils polarisent $22,176 \%$ de l'information, le plus souvent, l'essentiel de l'information est fourni par les deux premiers axes $\left(\mathrm{F}_{1}\right.$ et $\left.\mathrm{F}_{2}\right)$ d'où le choix porté sur eux pour effectuer notre analyse. L'axe $\mathrm{F}_{1}$ apporte $11,453 \%$ de l'information réelle et l'axe $\mathrm{F}_{2} 10,724 \%$.

Pour l'axe $\mathrm{F}_{1}$, la contribution des espèces varie de 0,001 à $23,432 \%$ et celle des relevés de 0,000 à $22,823 \%$ et l'axe $\mathrm{F}_{2}$, la contribution des espèces varie de 0,059 à $12,386 \%$ et celle des relevés de 0,005 à $7,331 \%$. Les espèces et/ou relevés ayant une contribution supérieure à la moyenne ont été utilisés pour la définition des axes $\mathrm{F}_{1}$ et $\mathrm{F}_{2}$. La Figure 2 donne la répartition spatiale des groupements végétaux dans les trois sites.

Dans les abscisses positives de $\mathrm{F}_{1}$, nous avons les relevés R57, R59, R60, R65, R66,
R67, R68 et R69 dans lesquels nous retrouvons les espèces à forte contribution comme Acacia penneta (L.) Willd, Celtis integrifolia Lam, Boscia senegalensis Lam, Commiphora africana (A.Rich.) Engl, Dichrostachys cinerea (L) et Grewia vilosa Willd. Ces relevés ont été effectués à Mont Rolland sur un sol argileux, par opposition aux relevés R6, R9, R10, R11, R12, R13, R25 et R27 dans lesquels nous trouvons des espèces comme Ziziphus mauritiana Lam, Combretum aculeatum Vent, Mangifera indica L, Piliostigma reticulatum (DC.), $B$. aethiopium, Calotropis procera Ait et Faidherbia albida (Del.) A. Che. Ces relevés qui se trouvent dans les abscisses négatives ont été réalisés à Fandène sur un sol sableux. L'axe $F_{1}$ oppose donc deux groupes de relevés différents par la texture, il pourrait donc constituer un gradient de texture.

En ce qui concerne l'axe $\mathrm{F}_{2}$, dans ses ordonnées positives, nous retrouvons les relevés dans lesquels sont présentes les espèces comme: Combretum micranthum G. Don, Anogeissus leiocarpus (DC) G. et perr, Acacia nilotica (L.) et Tamarindus indica L. Ce sont les relevés R34, R35, R40, R46 etc., qui ont été effectués à Sessène où la végétation est plus dégradée, par opposition aux relevés R57, R59, R60, R65, R66, R67, R68 et R69 qui ont été réalisés à Mont Rolland ou nous avons une végétation moins dégradée. Ces relevés à $A$. penneta, $C$. integrifolia, B. senegalensis, C. africana, D. cinerea et $G$. vilosa, se trouvent dans les coordonnées négatives de $F_{2}$. Ainsi l'axe $F_{2}$ pourrait correspondre à un gradient de dégradation. L'analyse factorielle de correspondance nous a permis de déterminer trois groupements: Le groupement à $A$. penneta (Gpt A.pen) retrouvé à Mont Rolland, $B$. aethiopium et $P$. reticulatum (Gpt B.eat et Pil.rec) à Fandène et le groupement à Guiera senegalensis J.F. Gmel et A. leiocarpus (Gpt B.eat et Pil.rec) retrouvé à Sessène. 


\section{Composition floristique des peuplements ligneux \\ Le peuplement ligneux de ces 3 sites} est riche de 35 espèces ligneuses réparties en 30 genres appartenant à 20 familles. La famille des Mimosaceae est la mieux représentée, suivie successivement de celle des Cesalpiniaceae, des Combretaceae et des Anacardiaceae (Tableau 2). Les autres familles ne sont représentées que par un seul genre. La répartition des espèces dans les genres montre que Acacia est le plus représenté avec 4 espèces suivi de Combretum (2 espèces).

La composition de la flore varie suivant les sites. Dans le site de Sessène, nous avons inventorié 25 espèces réparties en 22 genres et 17 familles. Les Mimosaceae et les Combretaceae sont les mieux représentées. La variabilité est plus importante avec les Mimosaceae et particulièrement dans le genre Acacia: 4 espèces à Mont Rolland, 3 espèces à Sessène et 2 espèces à Fandène. Dans la flore du site de Fandène, 22 espèces ont été inventoriées, réparties en 21 genres et 14 familles. La famille des Mimosaceae prédomine, suivie de celle des Combretaceae et des Caesalpiniaceae. Le site de Mont Rolland comporte 22 espèces appartenant à 19 genres et 14 familles. Les Mimosaceae sont les plus représentées, suivies des Capparaceae et des Caesalpiniaceae. Les autres familles ne sont représentées que par un seul genre et une seule espèce.

\section{Caractéristiques structurales du peuplement ligneux \\ -La densité}

Les densités des peuplements ligneux dans les 3 sites sont présentées dans le Tableau 3. Les densités, aussi bien réelle que théorique, varie d'un site à un autre. Ces densités sont élevées à Fandène et faibles à Sessène. Partout, la densité réelle est plus faible que la densité théorique. Le rapport entre les densités théorique et réelle montre que la densité réelle est environ 7,4 et 2 fois plus faible respectivement à Sessène, à Mont Rolland et à Fandène.

-La surface terrière

La surface terrière est de $13,86 \mathrm{~m}^{2} . \mathrm{ha}^{-1}$ à Sessène, 24,61 m².hà $\mathrm{m}^{-1}$ à Fandène et 49,64 $\mathrm{m}^{2} \cdot \mathrm{ha}^{-1}$ à Mont Rolland (Tableau 3). A Sessène, 2 espèces présentent une surface terrière importante. Il s'agit de $A$. digitata et F. albida. A Fandène, B. aethiopium, A. digitata et $F$. albida présentent une surface terrière élevée. Enfin à Mont Rolland, A digitata et $F$. albida ont une surface terrière importante.

\section{-Le recouvrement}

Le couvert ligneux varie de 5345,59 $\mathrm{m}^{2} \cdot \mathrm{ha}^{-1} \pm 84,39$ à Fandène, $1169,68 \mathrm{~m}^{2} \cdot \mathrm{ha}^{-1} \pm$ 54,77 à Mont Rolland et $1056,77 \mathrm{~m}^{2} \cdot \mathrm{ha}^{-1} \pm$ 72,73 pour le site de Sessène (Tableau 3 ).

Le recouvrement le plus important s'observe à Fandène et le plus faible à Sessène. A Fandène, les espèces qui ont un recouvrement très important sont: B. aethiopium, A. digitata, $F$. albida, Azadirachta indica L, $P$. reticulatum et $Z$. mauritiana. A Sessène, $F$. albida, Z. mauritiana, A. digitata et Gardenia ternifolia Schum. \& Thonn ont un recouvrement important. G. senegalensis, $F$. albida et $A$. digitata présentent un recouvrement important à Mont Rolland. -Répartition selon la circonférence

La répartition des individus selon la circonférence est présentée dans la Figure 3. A Fandène, la classe $75-100 \mathrm{~cm}$ renferme $27,19 \%$ des individus. Cette classe est suivie des classes $100-125 \mathrm{~cm}(17,05 \%)$ et $125-150$ $\mathrm{cm}(15,51 \%)$. Les classes inférieures à $75 \mathrm{~cm}$ renferment $26,21 \%$ des individus essentiellement constitués de B. aethiopium. Les individus de plus de $200 \mathrm{~cm}$ sont rares et représentent $11 \%$. La présence des jeunes individus montre une régénération relativement importante du peuplement dans ce site.

A Sessène, la répartition des individus par classes de circonférences montre que le peuplement est réparti dans presque toutes les classes de circonférences traduisant une 
certaine hétérogénéité du peuplement. La classe de $200 \mathrm{~cm}$ à $225 \mathrm{~cm}$ renferme $11,50 \%$ des individus, les plus petites classes $(<50$ $\mathrm{cm}$ ) renferment $12,40 \%$ des individus. Le peuplement de ce site est dominé par des espèces comme $F$. albida, $C$. micranthum, A. leiocarpus, A. nilotica et $T$. indica.

A Mont Rolland, la répartition des individus en fonction de la circonférence montre leur importance aux classes 10-25 et $25-50 \mathrm{~cm}(28,04 \%)$, ces classes sont dominées par des arbustes dont $A$. penneta et $B$. senegalensis. Le peuplement est réparti dans presque toutes les classes, mais contrairement à Sessène la fréquence des individus par classe dépasse rarement $5 \%$. Les individus dont la circonférence est supérieure à $500 \mathrm{~cm}$ représentent $20 \%$ du peuplement ligneux.

-Répartition selon la hauteur

La répartition des individus selon la hauteur est présentée dans la Figure 4. A Fandène, l'histogramme montre qu'environ $57 \%$ des individus ont des hauteurs comprises entre 0 et $7 \mathrm{~m}$. L'essentiel des individus observés dans ces classes est constitué de $B$. senegalensis et $A$. pennata. $41 \%$ des individus ont une hauteur comprise entre 7 et $14 \mathrm{~m}$, les espèces dominantes de ces classes sont $B$. aethiopium et $A$. digitata. Le peuplement ligneux est relativement dominé par les arbustes; les individus de plus de $14 \mathrm{~m}$ de hauteur ne représentent que $2 \%$ du peuplement.

A Sessène, les individus dont la hauteur est comprise entre 10,5 et $14 \mathrm{~m}$ (33\%) sont dominants. Les individus ayant une hauteur inférieure à $7 \mathrm{~m}$ représentent $39 \%$. Les arbustes sont essentiellement constitués par B. senegalensis, tandis que les arbres sont dominés par $B$. aethiopium et $A$. digitata.

A Mont Rolland, les individus du peuplement sont distribués dans toutes les classes de hauteur. La classe de hauteur de 0-3 m est la plus représentée avec $35 \%$ des individus. Elle est suivie de la classe $10-14 \mathrm{~m}$ puis $14-17 \mathrm{~m}$ qui constituent respectivement $16,10 \%$ et $13 \%$ du peuplement. Les individus de grande taille sont faiblement représentés avec des hauteurs comprises entre 24-28 m (6\%). Deux strates s'individualisent dans ce site, une strate arbustive $(41,5 \%)$ dominée par A. pennata et $B$. senegalensis et une strate arborée $(58,5 \%)$ dominée par A. digitata et B. aethiopium.

\section{Analyses de régression entre circonférence et hauteur}

La Figure 5 montre les relations de croissance entre la hauteur et la circonférence basale suivant les sites. Les coefficients de corrélations obtenus pour les trois sites entre la hauteur et la circonférence des arbres montrent des relations significatives entre la croissance en hauteur et celle en épaisseur. En effet, la valeur de ce coefficient est moyenne pour Fandène $\left(\mathrm{R}^{2}=0,537\right)$, soit $53 \%$, élevée pour Sessène et Mont Rolland avec respectivement $R^{2}=0,734$ et $R^{2}=0,739$, soit $73 \%$.

\section{Anthropisation du peuplement ligneux}

Dans nos relevés d'inventaire, des observations ont été faites sur l'exploitation des ligneux. Cette exploitation induit une réelle anthropisation des sites. L'exploitation est plus importante à Sessène avec un taux de $2,4 \%$. Le taux le plus faible a été enregistré à Fandène $(1,3 \%)$. Les coupes ont concerné essentiellement trois espèces: B. aethiopium, $F$. albida et $A$. digitata (Figure 6).

\section{Régénération du peuplement}

L'importance de la régénération en fonction des espèces est représentée par la Figure 7. En tenant compte des espèces, $B$. aethiopium $(29,79 \%)$ a le plus fort taux de régénération à Fandène, suivie de $B$. senegalensis $(20,85 \%)$ à Mont Rolland et de F. albida $(14,70 \%)$ à Sessène. Les espèces dominantes ont donc les taux de régénération les plus élevés. Elles représentent plus de $65 \%$ du taux global de régénération dans les trois sites.

Tableau 1 : Valeur propre et pourcentage de variance des quatre premiers axes de l'AFC. 


\begin{tabular}{lcccc}
\hline & $\mathbf{F}_{\mathbf{1}}$ & $\mathbf{F}_{\mathbf{2}}$ & $\mathbf{F}_{\mathbf{3}}$ & $\mathbf{F}_{\mathbf{4}}$ \\
\hline Valeur propre & 0,553 & 0,518 & 0,414 & 0,332 \\
\% variance & 11,453 & 10,724 & 8,577 & 6,877 \\
\% cumulé & 11,453 & 22,176 & 30,753 & 37,630 \\
\hline
\end{tabular}

Tableau 2 : Variation de la dominance relative $(\%)$ de quelques espèces inventoriées en fonction des différents sites.

\begin{tabular}{lcccc}
\hline Espèces & Peuplement & Sessène & Fandène & Mont Rolland \\
\hline B. aethiopium & 43,49 & 0,31 & 77,11 & 0,00 \\
B. senegalensis & 19,21 & 0 & 0,28 & 61,66 \\
F. albida & 17,26 & 49,24 & 15,28 & 7,69 \\
G. senegalensis & 6,86 & 42,51 & 2,42 & 0,25 \\
Z. mauritiana & 6,66 & 3,98 & 2,21 & 15,89 \\
A. digitata & 6,51 & 3,98 & 2,70 & 14,50 \\
\hline
\end{tabular}

Tableau 3 : Variation de quelques paramètres du peuplement en fonction des sites.

\begin{tabular}{|c|c|c|c|c|}
\hline $\begin{array}{l}\text { Paramètres } \\
\text { écologiques }\end{array}$ & $\begin{array}{l}\text { Densité réelle } \\
\text { (ind.ha }{ }^{-1} \text { ) }\end{array}$ & $\begin{array}{c}\text { Densité théorique } \\
\text { (ind.ha }^{-1} \text { ) }\end{array}$ & $\begin{array}{l}\text { Surface terrière } \\
\left(\mathbf{m}^{2} \cdot \mathbf{h a}^{-1}\right)\end{array}$ & $\begin{array}{c}\text { Recouvrement } \\
\left(\mathbf{m}^{2} \cdot \mathrm{ha}^{-1}\right)\end{array}$ \\
\hline Sessène & 19 & 138 & 13,86 & 1056,77 \\
\hline Ecart-type & 1,71 & 12,22 & 1,69 & 72,73 \\
\hline Fandène & 109 & 244 & 24,61 & 5345,59 \\
\hline Ecart-type & 17,14 & 5,26 & 2,79 & 84,39 \\
\hline Mont Rolland & 37 & 153 & 49,64 & 1169,68 \\
\hline Ecart-type & 5,69 & 7,99 & 11,80 & 54,77 \\
\hline
\end{tabular}

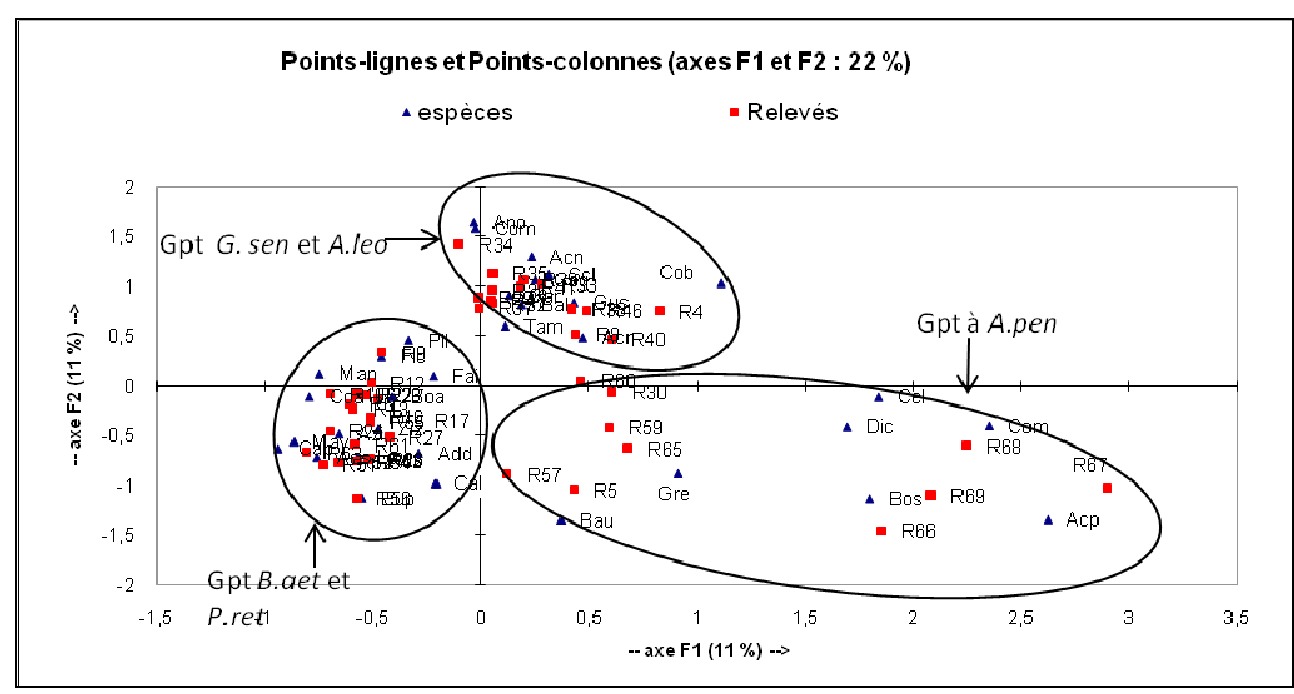

Figure 2: Diagramme de la matrice 69 relevés x 35 espèces ligneuses dans les trois sites d'étude. 
Fandene

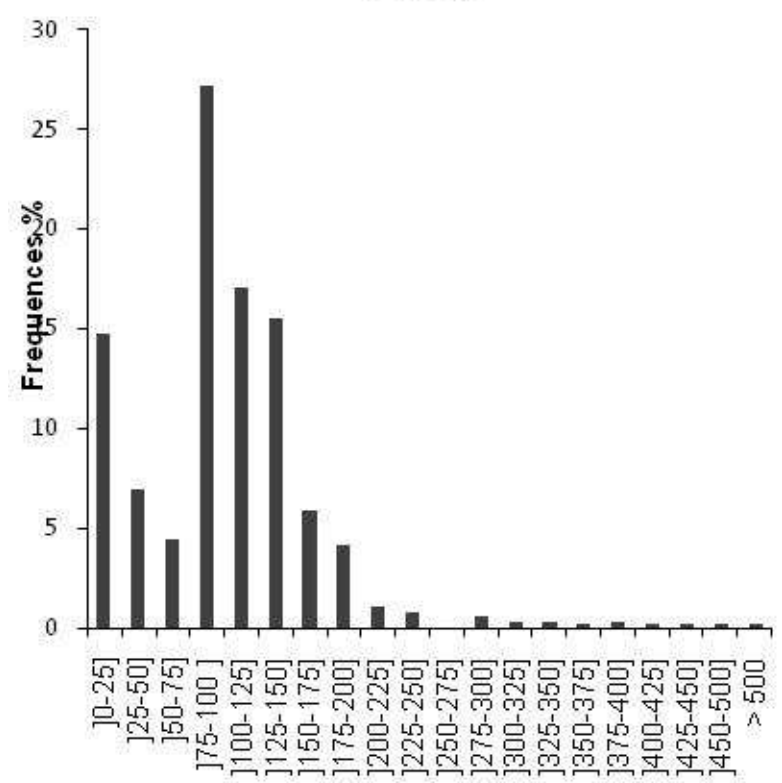

Classe de circonferences $(\mathrm{cm})$

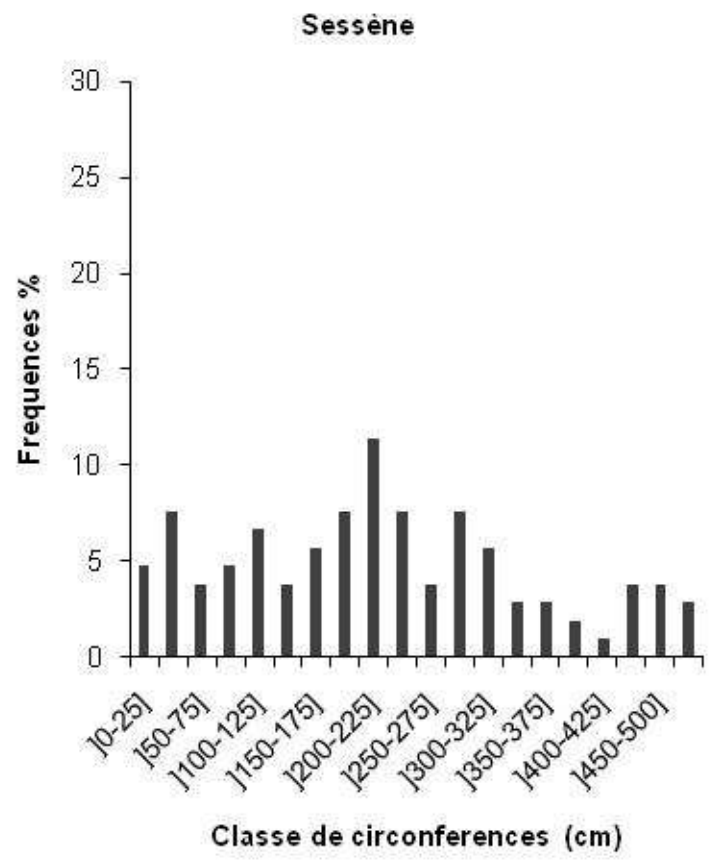




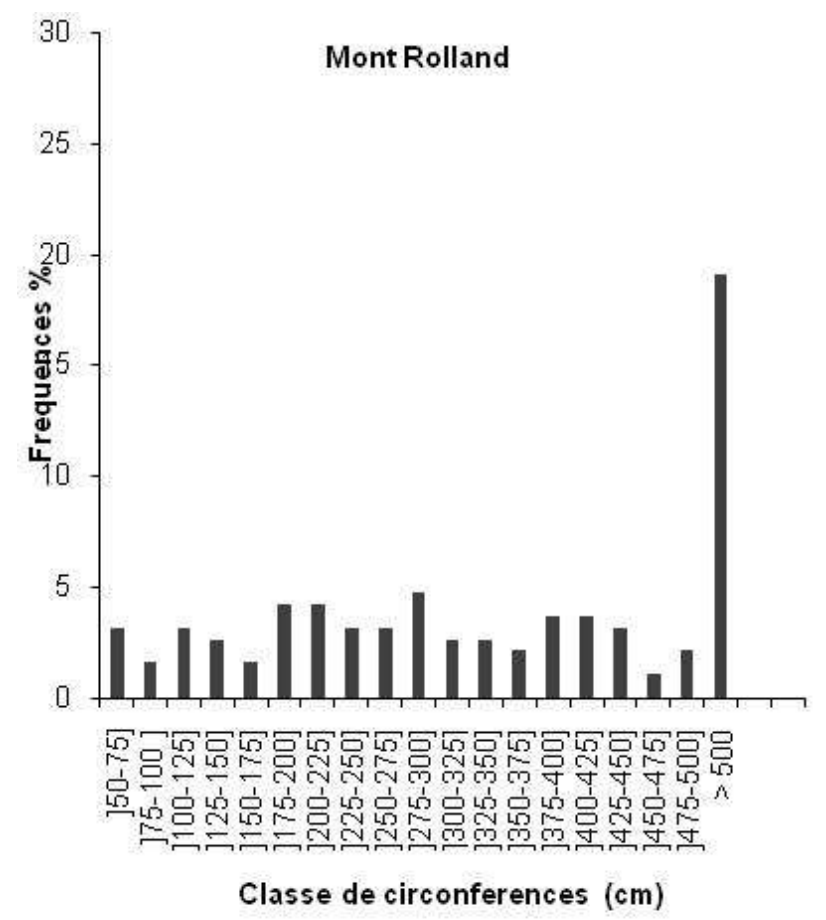

Figure 3 : Répartition des fréquences $(\%)$ de la végétation ligneuse par classes de circonférences (cm) dans les trois sites.

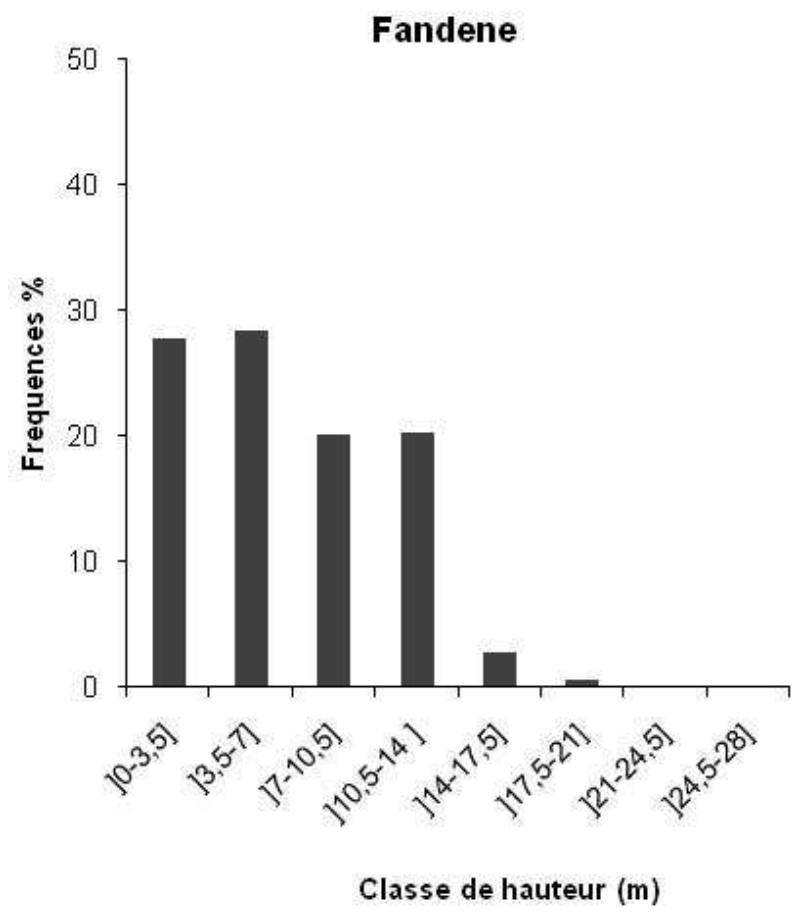




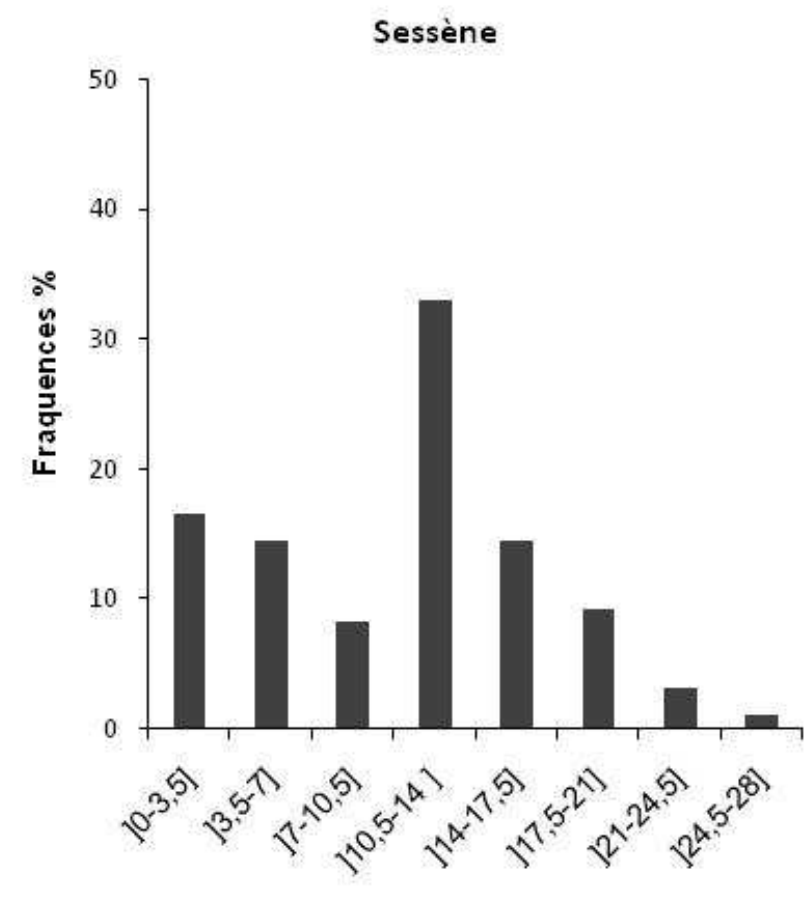

Classe de hauteur (m)

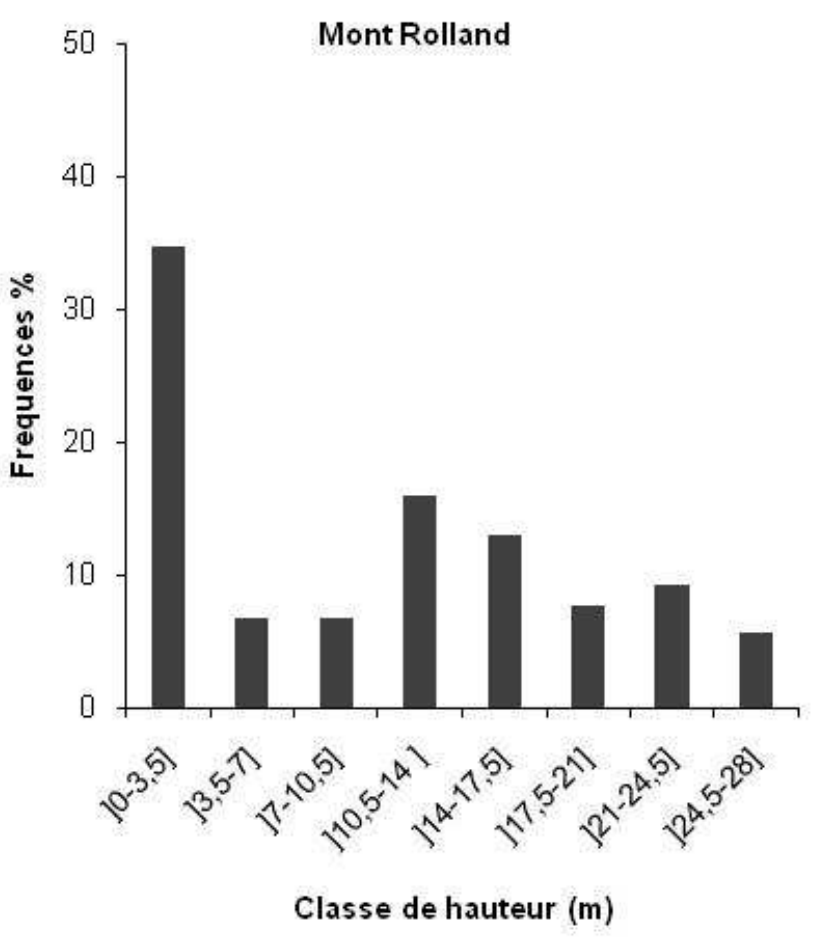

Figure 4 : Répartition des fréquences (\%) de la végétation ligneuse par classes de hauteur (m) dans les trois sites. 


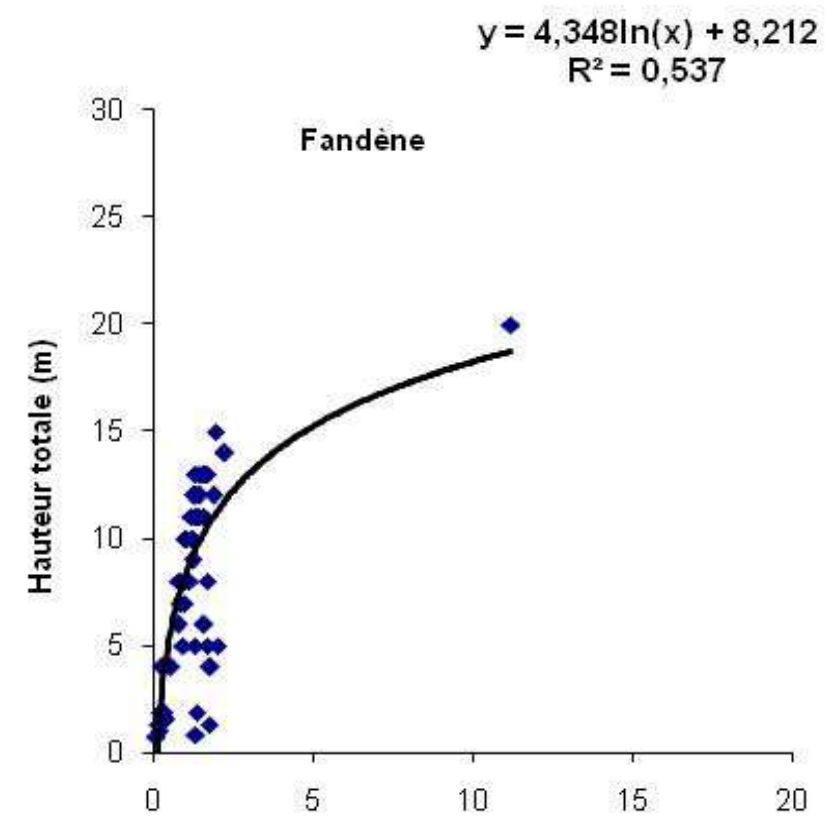

Circonférence à $0,30 \mathrm{~m}$ du sol (m)

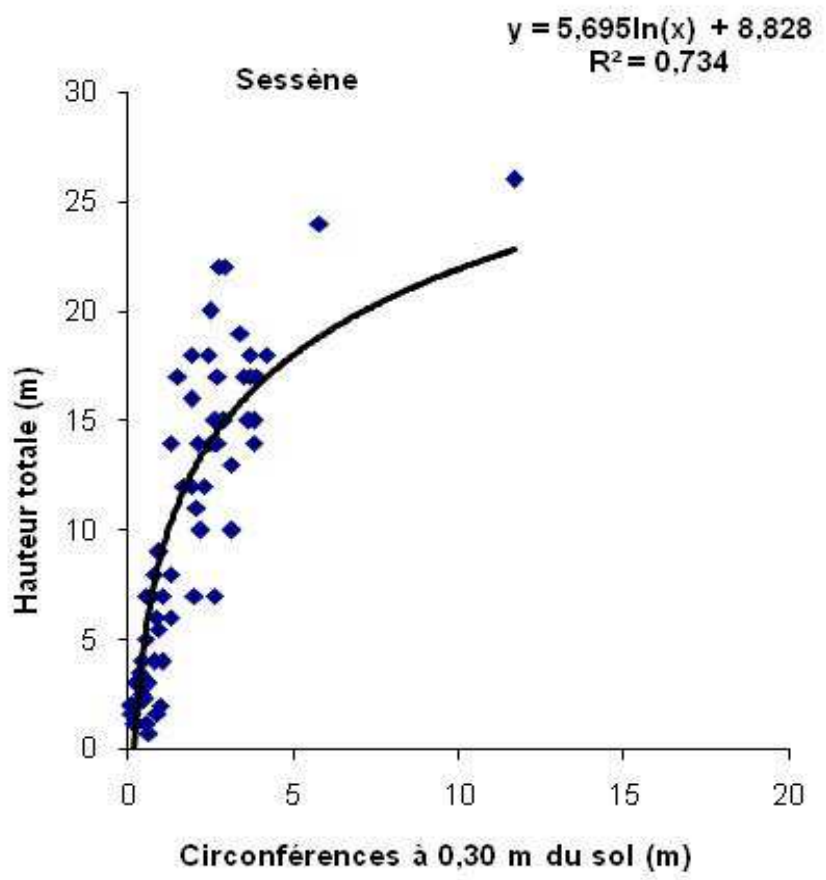




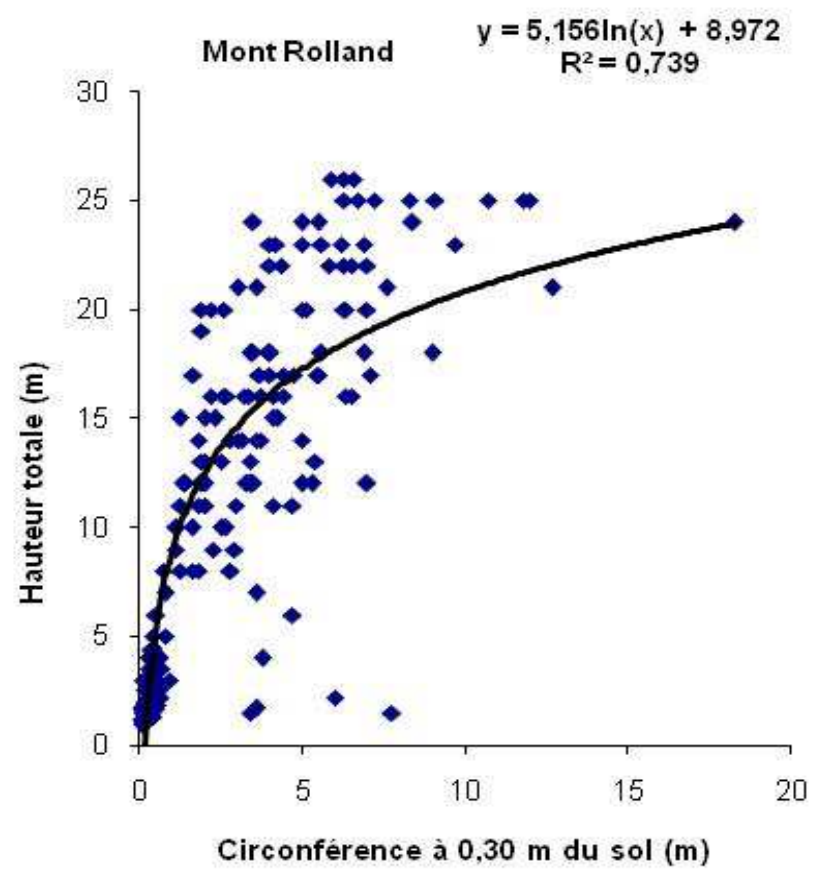

Figure 5 : Relation entre la hauteur et la circonférence de la végétation ligneuse dans les trois sites.

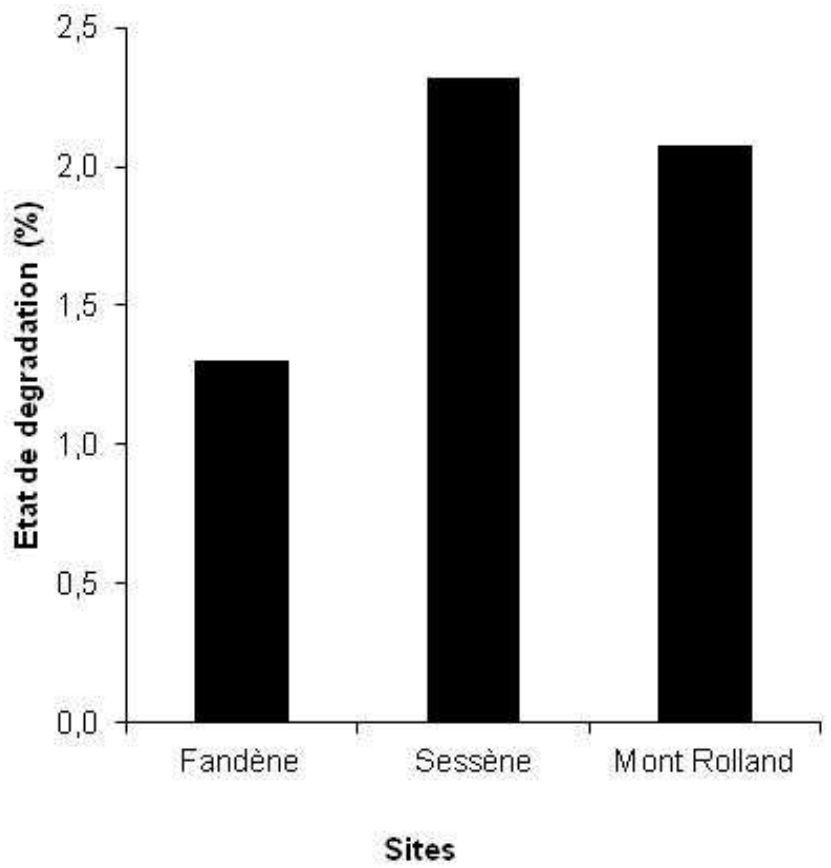

Figure 6: Taux d'exploitation de trois espèces ligneuses (B. aethiopium, F. albida et A. digitata) dans les trois sites. 


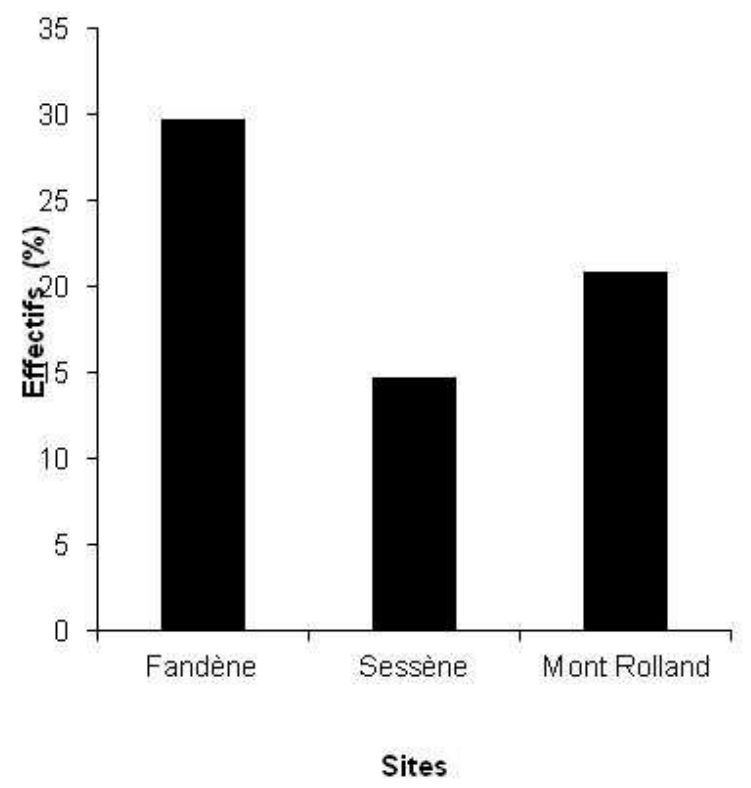

Figure 7 : Distribution des jeunes plants de trois espèces ligneuses (B. aethiopium, F. albida et A. digitata) dans les trois sites.

\section{DISCUSSION}

L'analyse

factorielle de correspondance a permis de distinguer trois groupements végétaux qui sont liés aux variations des conditions du milieu.

Le groupement à $A$. penneta, retrouvé à Mont Rolland, est caractérisé par des sols argileux et riches en matière organique. Cette espèce joue un rôle important dans l'amélioration des sols (Maydell, 1983).

Le groupement à $B$. aethiopium et $P$. reticulatum à Fandène est caractérisé par des sols sableux. B. aethiopium (espèce caractéristique du site) joue un rôle dans la protection des terres agricoles et dans l'alimentation. Quant à $P$. reticulatum, elle est souvent laissée dans les champs par les populations du Bassin arachidier pour son bois de chauffe.

Le groupement à $G$. senegalensis et $A$. leiocarpus est rencontré à Sessène. Les sols sur lesquels s'est installé ce groupement sont très dégradés. G. senegalensis est une espèce caractéristique de ce groupement. Charhabil (2006) a montré que cette espèce résiste aux actions anthropiques (Bois de chauffe). Quant à A. leiocarpus, Bellefontaine (2005) souligne que cette espèce régénère bien dans les systèmes d'utilisation des terres de cette zone du bassin arachidier.

Au cours de notre étude, nous avons recensé 35 espèces réparties en 30 genres et 20 familles. L'analyse de la présence ou de l'absence des espèces dans les différents sites a montré une variation inter-site. Le site de Sessène renferme 25 espèces alors que les sites de Fandène et Mont Rolland enregistrent chacun 22 espèces. De façon générale, les espèces dominantes sont $B$. aethiopium, $F$. albiba et $A$. digitata.

À Fandène et à Sessène, les espèces dominantes sont respectivement $B$. aethiopium et $F$. albiba. Cette dominance s'expliquerait par leurs adaptations aux conditions 
édaphiques. En effet, B. aethiopium pousse sur un sol à faible pouvoir de rétention d'eau voire sur tous les types de sols et elle s'adapte à la sécheresse comme à l'inondation. Quant à $F$. albiba, c'est une essence forestière fréquemment utilisée au sein des terroirs du bassin arachidier et sa litière a montré un effet positif sur tous les paramètres de croissance (Diallo et Guissé, 2008).

Outre ces deux espèces, $B$. senegalensis, Z. mauritiana et A. digitata dominent à Mont Rolland du fait de l'adaptation de l'espèce à la sécheresse et aux sols argileux (INP, 2009).

La surface terrière est plus élevée à Mont Rolland par rapport à Fandène et à Sessène. Malgré sa densité relativement faible, Mont Rolland présente des individus à gros diamètre contrairement à Fandène où la densité est élevée avec une surface terrière faible. En effet, au niveau de ces trois sites, les densités théoriques sont supérieures aux densités réelles suggérant une distribution irrégulière des individus (Canard et al., 2004).

Le recouvrement des ligneux observé à Fandène est très important de même que la densité des arbres, par contre à Mont Rolland ce recouvrement est faible par rapport à Fandène malgré la forte présence d'individus à gros diamètres. Cette baisse du recouvrement observée à Mont Rolland s'accentue à Sessène à cause des activités anthropiques. En effet dans cette localité, la population n'épargne pas des arbres qui ont un intérêt socio-économique (Thione, 2000) tels que $B$. aethiopium et $A$. digitata.

A Fandène et à Mont Rolland, il y a eu beaucoup plus de régénération avec les densités des jeunes plants plus élevées contrairement à Sessène à cause des activités anthropiques intenses. En effet, les jeunes individus seraient éliminés pendant les travaux champêtres (Thiaw, 2009).
La distribution des individus dans les classes de hauteur montre une forte proportion dans la classe de 0-3,5 m à Mont Rolland et à Fandène par opposition à Sessène. Cela montre une meilleure régénération dans les 2 premiers sites. Cette observation est confirmée par la structure du peuplement par classes de circonférence qui indique une bonne régénération à Mont Rolland suivi de Fandène et Sessène.

Les analyses de régression montrent une liaison étroite entre la circonférence et la hauteur. Cependant, les hauteurs ne suivent pas les mêmes variations que les circonférences et la liaison se trouve sujette à de nombreux facteurs plus particulièrement stationnels et sylvicoles. En effet, la hauteur ne croît pas indéfiniment en fonction du diamètre. La croissance en hauteur des ligneux se stabilise autour de $25 \mathrm{~m}$, alors que le diamètre peut continuer à croître. Ces résultats corroborent les travaux de Adjonou et al. (2009) dans les forêts claires du parc national Oti-Keran au Nord-Togo sur la structure, dynamique et impacts des modifications climatiques récentes.

L'anthropisation du peuplement ligneux la plus importante a été observée à Sessène et la plus faible à Fandène. A Sessène, elle pourrait être liée aux contraintes du milieu telles que la pression croissante du bétail. Les espèces concernées par cette exploitation sont $F$. albida à Sessène, $B$. aethiopium à Fandène et $A$. digitata à Mont Rolland. Cette anthropisation entrave considérablement les processus naturels d'évolution et de renouvellement de cet écosystème (Ngom, 2008).

\section{Conclusion}

Ce travail nous a permis de mettre en évidence la structure et le potentiel de régénération des peuplements ligneux, de déterminer les groupements végétaux qui sont 
liés aux conditions édaphiques du milieu et le degré d'anthropisation du milieu. La flore et la végétation ligneuses de la région de Thiès sont nettement dominées par les Mimosaceae et les Combretaceae. L'analyse de l'importance spécifique a révélé en outre une dominance nette de $B$. aethiopium, $B$. senegalensis, F. Albida, G. senegalensis, A. digitata et $Z$. mauritiana. Ces dernières espèces présentent aussi de très nombreux rejets. La distribution des individus dans les classes de hauteur fait ressortir que les individus de grande taille sont faiblement représentés dans les trois sites, quant à la circonférence, le peuplement est réparti dans presque toutes les classes traduisant une certaine hétérogénéité du peuplement dans les sites de Sessène et Mont Rolland.

Il nous paraît important de poursuivre les investigations par des enquêtes ethnobotaniques auprès des populations afin de mieux connaître leurs préoccupations et les différentes utilisations des espèces (feuilles, fruits, racines et écorces) et les pratiques influençant la pauvreté des sols.

\section{REFERENCES}

Adjonou K, Bellefontaine R, Kokou K. 2009. Les forêts claires du Parc national OtiKeran au Nord-Togo: structure, dynamique et impacts des modifications climatiques récentes. Sécheresse, 20(1e): e1-e10.

Aubréville A. 1962. Position Chronologique du Gabon. Flore du Gabon. Muséum Hist. Nat. : Paris ; 3-11.

Bakhoum C. 2012. Diversité et capacité de régénération naturelle du peuplement ligneux dans les systèmes agraires de bassin arachidier en zone soudanosahélienne (région de Kaffrine, Sénégal). Mémoire de Thèse de Doctorat en Biologie végétale, Option Ecologie, FST. UCAD, 155p.
Bellefontaine R. 2005. Régénération naturelle à faible coût dans le cadre de l'aménagement forestier en zones Tropicales sèches en Afrique. Vertigo, La Revue en Sciences de l'Environnement, 6(2) : 15p.

Berhaut J. 1967. Flore du Sénégal. Clairafrique éd. : Dakar ; 485p.

Canard A, Poinsot D. 2004. Quelques méthodes statistiques typiques de l'étude des populations et des peuplements par la méthode des quadrats. Fiche technique, Université de Renne1, 34p.

Centre de suivi écologique. 2009. Plan régional d'action pour l'Environnement. Ministère de l'Environnement et de la Protection de la Nature, Dakar, 39p.

Charahabil MM. 2006. Flore et végétation ligneuses de trois forêts communautaires $\mathrm{du}$ Sine-Saloum (Centre-Ouest du Sénégal). Mémoire DEA, FST, UCAD (Sénégal), 27 p.

Diallo A, Madiara NF, Guissé A. 2011. Structure des peuplements ligneux dans les plantations d'Acacia sénégal (L.) Willd dans la zone de Dahra (Ferlo, Sénégal). Rev. Écol. (Terre Vie), 66 : $415-427$.

Diallo D, Guissé A. 2008. Influence de la litière foliaire de cinq espèces végétales tropicales sur la croissance du mil (Pennisetum glaucum (L.) R. BR.) et du maïs (Zea mays L.), 5p.

INP. 2009. Institut national de pédologie. Compte rendu de mission dans le zone pédoclimatique centre nord du Sénégal (Thiès), 17p.

Minda MS. 2009. Contribution à la caractérisation bio pédoclimatique de la zone Cayor Baol (région de Thiès) au Sénégal. Mém de DEA, UCAD, 50p.

Lebrun JP, Stork A. 1997. Enumération des Plantes à Fleurs d'Afrique Tropicale (vol 4). Edition des Conservatoires et 
Jardin Botaniques de la Ville de Genève : Genève ; 711p.

Matheron G. 1973. The intrinsic random functions and their applications. $A d v$. Appl. Prob., 5: 439-468.

Maydell HJV. 1983. Arbres et Arbustes du Sahel. Office Allemand de la coopération technique, GTZ, Eschborn : Allemagne ; 531.

Ndiaye O. 2009. Contribution à la caractérisation Biodepoclimatique des Niayes de Pikine et de Saint Louis. Mémoire de DEA, Université Cheikh Anta Diop, Dakar, 68p.

Ngom D. 2008. Définition d'indicateurs de gestion durable des ressources sylvopastorales au Ferlo (Nord-Sénégal). Thèse de Doctorat de $3^{\text {eme }}$ Cycle en Biologie végétale, Option Ecologie, FST, UCAD, 148p.
Rondeux J. 1993. La Mesure des Arbres et des Peuplements Forestiers. Les Presses Agronomiques de Gembloux : Paris ; 16 et 59 .

Thiaw A. 2009. Contribution à la Caractérisation Biodepoclimatique de la région de Kaffrine (zone centre-ouestSénégal). Mémoire de DEA, Université. Cheikh Anta Diop, Dakar, 58p.

Thione LA. 2000. Biologie de la reproduction et étude de l'impact de l'exploitation des feuilles et des fruits sur la productivité des rôniers. Doctorat de 3eme cycle, l'Université Cheikh Anta Diop, Faculté des sciences et techniques, Département de Biologie Végétale, Dakar, 138p.

Wouters P, Notelaers V. 1999. L'espace entre les arbres et la densité des peuplements. Sylva Belgica, 106(21) : 4 p. 